\title{
Perspectives on sensory processing disorder: a call for translational research
}

\author{
Lucy J. Miller 1,2,3*, Darci M. Nielsen', Sarah A. Schoen ${ }^{1,2,3}$ and Barbara A. Brett-Green ${ }^{1,2}$ \\ 1 Sensory Processing Disorder Foundation, Greenwood Village, CO, USA \\ 2 University of Colorado at Denver, Denver, CO, USA \\ 3 Rocky Mountain University of Health Professionals, Provo, UT, USA
}

\section{Edited by:}

John J. Foxe, Nathan S. Kline Institute for Psychiatric Research, USA; City

College of the City

University of New York, USA

Reviewed by:

David J. Lewkowicz, Florida Atlantic University, USA

John G. McHaffie, University of Wake

Forest, USA

\section{*Correspondence:}

Lucy J. Miller, Sensory Processing

Disorder Foundation, 5655 South

Yosemite Street Suite 305, Greenwood

Village, CO 80111, USA.

e-mail:miller@SPDfoundation.net
This article explores the convergence of two fields, which have similar theoretical origins: a clinical field originally known as sensory integration and a branch of neuroscience that conducts research in an area also called sensory integration. Clinically, the term was used to identify a pattern of dysfunction in children and adults, as well as a related theory, assessment, and treatment method for children who have atypical responses to ordinary sensory stimulation. Currently the term for the disorder is sensory processing disorder (SPD). In neuroscience, the term sensory integration refers to converging information in the brain from one or more sensory domains. A recent subspecialty in neuroscience labeled multisensory integration (MSI) refers to the neural process that occurs when sensory input from two or more different sensory modalities converge. Understanding the specific meanings of the term sensory integration intended by the clinical and neuroscience fields and the term MSI in neuroscience is critical. A translational research approach would improve exploration of crucial research questions in both the basic science and clinical science. Refinement of the conceptual model of the disorder and the related treatment approach would help prioritize which specific hypotheses should be studied in both the clinical and neuroscience fields. The issue is how we can facilitate a translational approach between researchers in the two fields. Multidisciplinary, collaborative studies would increase knowledge of brain function and could make a significant contribution to alleviating the impairments of individuals with SPD and their families.

Keywords: multisensory integration, sensory integration, sensory processing disorder, translational research

\section{INTRODUCTION}

This article explores the convergence of two fields: a clinical field originally known as sensory integration, and a branch of neuroscience that also uses the term sensory integration to describe specific studies of sensation. Clinically, the term sensory integration was first used by Ayres (1966b, 1972b) to identify a field of study related to individuals with atypical responses to sensory stimulation. The clinical condition is now known as sensory processing disorder (SPD). In neuroscience, early sensory system research emphasized unisensory studies. With the advent of technology a new branch of neuroscience research focuses on multisensory integration (MSI), which studies the interaction of two or more sensory modalities.

Early neuroscience research on the interaction of individual sensory systems provided a foundation for Ayres to develop clinical hypotheses about the disorder and the treatment. The clinical identification of SPD in children was further based on the applied research and empirical observations of children with learning disabilities made by Ayres in the 1960s-1980s (Ayres, 1966b, 1972b). Ayres was an occupational therapist and researcher who completed a post-doctoral fellowship at the Brain Research Institute at UCLA. She conducted numerous research studies, the beginning of empirical science for this new clinical disorder, which she hypothesized related to neurological impairment in detecting, modulating, discriminating and responding to sensory information (Ayres, 1965, 1966a, 1972b). Ayres labeled the theory sensory integration theory, the assessments she developed tests of sensory integration, the clinical disorder sensory integration dysfunction, and the treatment she founded sensory integration treatment.

Because the clinical and the neuroscience fields both use the term sensory integration, understanding the specific meanings intended by each field is critical. This article focuses on clarifying the construct of sensory integration as used by the clinical field and how translational research could elucidate the underlying neural mechanisms, objectify the diagnostic criteria, and support the evaluation of treatment effectiveness. Collaboration between clinical and basic sciences has the potential to improve the quality of life for those with SPD and their families, as well as provide insight into central nervous system functioning.

\section{CLINICAL FIELD RELATED TO SENSORY INTEGRATION DESCRIPTION OF THE DISORDER}

Sensory processing disorder is a heterogeneous condition that includes a variety of subtypes. Individuals with the disorder have impaired responses to, processing of, and/or organization of sensory information that effects participation in functional daily life routines and activities. Although the clinical field is not completely unified in how to define the subtypes of SPD, a new nosology hypothesizes six subtypes (Miller, 2006; Miller et al., 2007a). Recent feature analysis and mathematical modeling sug- 
gests these subtypes are discreet, although individuals may present with a combination of subtypes (R. Picard and E. Hedman, personal communication).

The new nosology proposes three main categories within SPD: sensory modulation disorder (SMD), sensory discrimination disorder and sensory-based motor disorder. SMD refers to difficulty regulating responses to sensory stimulation and three subtypes are proposed: Sensory over-responsive (responds too much, for too long, or to stimuli of weak intensity); Sensory under-responsive (responds too little, or needs extremely strong stimulation to become aware of the stimulus); and sensory seeking/craving (responds with intense searching for more or stronger stimulation). All three modulation subtypes have in common difficulty grading or regulating responses to sensory stimuli. Within sensorybased motor disorder two subtypes are proposed: Postural disorder, which reflects problems in balance and core stability, and dyspraxia, which encompasses difficulties in motor planning and sequencing movements. Sensory discrimination disorder refers to difficulty interpreting the specific characteristics of sensory stimuli (e.g., the intensity, the duration, the spatial, and the temporal elements of sensations; Miller, 2006; Miller et al., 2007a). Sensory discrimination disorder can be present in any of the seven sensory systems (i.e., vestibular, proprioceptive, and the five basic senses).

\section{DESCRIPTION OF THE INTERVENTION}

Sensory integration treatment, as originally conceptualized by Ayres (1972a) involves the use of multisensory environments where challenging goal-directed activities are designed to provide specific sensory input. The child is supported to display appropriate responses and behavior during the therapist-guided activity. Through repetition the child's brain begins to process sensory stimulation more normally and he/she begins to interact effectively within sensory environments (Ayres, 1958). A constant "upping the ante" and use of the therapeutic relationship to scaffold the child with each new challenge is the key to progress. Techniques use sensory stimuli and cognitive strategies to assist the child in attaining and maintaining a regulated state, sustaining attention, controlling emotions and behaviors, as well as completing complex motor skills. Activities are designed to be "fun" and tap the inner drive of the child, incorporating sensory input such as swinging (vestibular), jumping, pulling, pushing (proprioceptive) and deep pressure touch (tactile) as well as visual, auditory, olfactory, and gustatory input. Repetition of normal responses to sensory stimuli is hypothesized to create new neural pathways (Hebb, 1949) and provide the platform for successful participation in natural realworld environments (Ayres, 1958).

\section{EARLY CLINICAL FIELD}

Sensory integration dysfunction/SPD was first identified as a clinical condition by Ayres (1972a). Ayres defined sensory integration as "the organization of sensory information for use" (Ayres, 1979), with "use" referring to behaviors, motor abilities, and other complex functional responses produced after sensory input. Ayres' meaning of sensory integration was related to the ability to process information from multiple sensory modalities during daily routines and activities (e.g., enjoying walking barefoot on the beach, riding a bicycle). Thus, sensory integration as described by occupational therapists is critical to one's ability to perform daily life activities, such as getting dressed in the morning, playing on playground equipment, participating in team sports or attending a birthday party. Often problems in the clinical condition manifest as emotional and behavioral symptoms including anxiety, aggression, and inattention (Ayres, 1963).

Ayres focused her theory on children with learning disabilities, but noted that the constructs might also apply to other conditions (Ayres and Tickle, 1980). Since her passing in 1988, clinicians have used sensory integration therapy with a wide variety of disorders. However, treatment research prior to 1999 was widely criticized for lack of methodological rigor, both within the occupational therapy field where it originated (Polatajko et al., 1992; Wilson et al., 1992; Vargas and Camilli, 1999) and outside the field (Taft, 1972; Denhoff, 1981; Schaffer, 1984; Arendt et al., 1988). After Ayres' original writings, and before 1999, strong empirical evidence related to the validity of the condition and effectiveness of the treatment approach was not available in peer-reviewed literature (Miller et al., 2007a).

\section{CONSTRUCTS RELATED TO THE CLINICAL THEORY}

Ayres' theories and the theoretical underpinnings related to MSI in neuroscience are, to a large extent, based on the same early neuroscience research. Most prescient, Ayres endorsed the idea that single system sensory research was not reflective of the way the brain functions. Her theory was built on the belief that the sensory systems interact almost instantaneously to make sense of every experience, which requires subconscious integration of information from more than one sensory system (Ayres, 1958, 1961). "Sensory integration sorts, orders, and eventually puts all of the individual sensory inputs together into whole brain function" (Ayres, 1979, p. 28).

A brief review of Ayres' theory, based on the neuroscience doctrine of her time, suggests five primary constructs of her theory, three related to the diagnosis and two related to the intervention. Her theoretical synthesis was formulated on a basic assumption that the brain function of children with SPD was not normal and that by understanding the child's underlying deficits, appropriate treatment approaches could be designed (Ayres, 1975). Additionally, in developing her intervention model, she hoped to improve the knowledge base related to understanding the complex interweaving of multiple sensory functions essential to growth, development and learning.

\section{CONSTRUCTS RELATED TO THE DISORDER Brain functions as a whole}

Ayres based her hypotheses about SPD on principles of normal development. Citing Luria (1966), she endorsed the premise that the overlapping sensory topology of the brain was structured to produce efficient functioning, with all structures being inter-related and interaction being facilitated through the brain's association areas. She believed that all neurons communicated with all other neurons so that the brain functioned as a whole (Ayres, 1961) and that nervous system function would be efficient. Ayres conceptualized the underlying deficit in SPD as inefficient functioning of the central nervous system.

\section{Importance of sequential development}

Three primary neurobiological percepts guided Ayres' thinking about SPD: (1) development follows a predictable sequence; (2) 
abnormal development may reflect the expression of more primitive behavior and (3) maturation is dependent on interaction with the environment. Using theories of Piaget (1952), and Ames and Ilg (1964), Ayres described how the sequential pattern of increasingly more complex motor behavior provides a marker of the maturation of the central nervous system. Ayres supported the premise that each evolutionary progression incorporates new reorganized behaviors citing Green's (1958) study of successive levels of complexity in the brain of lower vertebrates. She suggested that SPD is a deviation from normal development, with observable behaviors that are reflective of lower levels of function (Ayres, 1954, 1961, 1966a). For example, Ayres emphasized that the gradual ability to inhibit primitive affective responses to sensory experiences, allows one to develop the ability to discriminate higher-level aspects of the stimuli such as spatial and temporal characteristics. Children with the sensory over-responsive subtype of SPD were hypothesized to be stuck at this primitive stage of development based on the observation of fight or flight behaviors in response to normal sensory experiences (Ayres, 1963). Ayres' theory highlighted that developmental phases are "pre-programmed" based on phylogeny (Ayres, 1966a), after which the individual's ontogeny (e.g., an interaction of environment and genes) produces the individual's unique characteristics (Ayres, 1954, 1975). Based on the research of Scheibel and Scheibel (1964), Ayres noted that in development, the older parts of the brain become more firmly connected and interactions among existing neural structures are established. Her synthesis of the neurobiological studies of the day was used to support her premise that normal development reflects the interaction between one's innate abilities and environmental opportunities (Ayres, 1975).

\section{Etiologic factors}

Ayres discussed both environmental and genetic factors as proposed etiologies for SPD. She noted that symptoms reported in those who had experienced sensory deprivation were similar to symptoms observed in children with sensory processing (SP) issues. For example, she cited studies that when deprived of sensation, structural neural changes occur (Levin and Alpert, 1959; Melzack, 1962) and the brain generates its own input, resulting in hallucinations and distortions (Solomon et al., 1961). In addition, she hypothesized that "genetic factors in certain children may make one part of the brain more vulnerable than usual. In this highly vulnerable state, environmental toxins [or prenatal stressors] may interfere with sensory integrative development" (Ayres, 1979, p. 54).

\section{CONSTRUCTS RELATED TO TREATMENT}

\section{Role of plasticity, active participation and environment}

Ayres' intervention model was predicated on three basic neural principles: neural plasticity makes brain change possible, active participation is required for neural changes, and enriched environments are needed to guide neural changes (Ayres, 1972b). She linked the principle of neural plasticity to studies of Schiebel and Schiebel (1964) hypothesizing that the changes observed in treatment might have resulted from increases in dendritic growth and greater potential for learning (Ayres, 1972c). Her intervention model required the client to engage actively in challenging tasks in an enhanced multisensory environment. This tenet was based on multiple studies that showed animals in enriched, environments developed increased cortical weight and better problem solving abilities than animals passively exposed to sensation (Bennett et al., 1964; Rosenzweig, 1966; Dru et al., 1975). Ayres cited studies demonstrating that repeated active challenges paired with successful responses promote central nervous system growth and that cognitive abilities grow from organizing incoming sensory input (Lassek, 1957). Furthermore, she noted that active participation was supported by research that showed biochemical changes from engagement in meaningful trial-and-error learning during a sensory and motor task (Hyden and Egyhazi, 1962).

\section{Importance of multisensory stimulation and the role of challenge and success}

Ayres also based her intervention model on two key principles: activities must use more than one sensory system concurrently (Ayres, 1975) and activities must provide the "just right challenge" (Ayres, 1979). Her ideas about using more than one sensory system to increase learning were based on the findings of Jung et al. (1963). They concluded that multisensory stimulation is often more effective than unisensory stimulation in changing behavior based on the finding in cats that some cells in the thalamus are multisensory, often responding to sound, sight and touch/pressure. Citing Herrick (1956), Ayres theorized that neural mechanisms were reverberating feedback and feed forward systems, designed to translate afferent information into action. She stressed that although neural activity is constant and disorganized, typically developing individuals automatically sort and organize messages from many sensory neurons before sending information on to motor neurons (Ayres, 1958). This allows the person to stay regulated and organize actions.

Ayres also observed that tasks, which are both challenging and to which a successful response is made have a particularly strong integrating effect. Therefore in treatment sessions, therapists guide the child to perform challenging tasks and support the child to be successful. Usually, the response is a motor action after sensory input. Ayres supported her premise by the work of Buser and Imbert (1961), who found that the greatest converging intersensory integration is located in the motor cortex with additional sensory convergence in the premotor cortex. This suggests a greater likelihood of stimulating intersensory integration with gross motor compared to fine motor movements. Building on Sherrington's work (Sherrington, 1906, 1955), Ayres described sensory integration as "the interaction and coordination of two or more functions or processes in a manner which enhances the adaptiveness of the brain's response" (Ayres, 1972b, p. 25-26) and the ability to respond with "directed flexibility" (Ayres, 1972b, p. 26).

\section{A CALL FOR THEORY ADVANCEMENT}

Ayres, trained as a neuroscientist, knew that her theories would change as new empirical evidence became available. In fact, a hallmark of Ayres' lifetime of research was to constantly revise her theory and generate new hypotheses based on new clinical and neuroscience research. The clinical theoretical framework that guided Ayres' work was based on a thorough review and synthesis of the neuroscience literature of her generation (1960-1988). However, after Ayres passed away, empirical science supporting her 
theories was slow to progress. Notable was the lack of advances in establishing either the etiological precursors or the neurobiological substrates of SPD.

\section{CURRENT CLINICAL RESEARCH}

Since 1995, empirical evidence related to the validity of SPD as a separate syndrome and research methods evaluating the effectiveness of the treatment approach have become more rigorous (Miller et al., 2007a). A spurt of developmental psychobiology research occurred beginning in 1995, due to the interest of and funding from the Wallace Research Foundation. This research advanced SPD knowledge and increased awareness and recognition of SPD as a novel diagnostic entity. In fact, the evidence is so compelling that the Diagnostic and Statistical Manual (DSM)-V revision committee is currently considering the inclusion of SPD into the "novel diagnoses" section of the DSM-V.

Much of the new research is derived from a SPD Scientific Work Group (SWG) of NIH-funded scientists who had programs of research in etiology, genetics, neurobiology or treatment effectiveness and who agreed to add SPD to their current study of other neurobiological disorders. The SPD-SWG used Pennington's model of syndrome validation (Pennington, 1991) as a blueprint to identify key areas of research required to determine if SPD was a valid syndrome. Pennington (1991) stated that the more divergence shown with existing disorders the more likely it was that a newly conceptualized condition could be considered a unique syndrome. The subtype that has been most studied by the SPD-SWG is SMD, particularly sensory over-responsivity (SOR) and sensory underresponsivity (SUR) subtypes. The recent findings of the SPD-SWG described below are divided into two sections: (1) research related to the disorder, including a brief discussion of etiology, signs and symptoms, prevalence and neural features and (2) research related to the treatment, which includes new research on treatment effectiveness using Ayres' intervention model.

\section{RESEARCH RELATED TO THE DISORDER \\ Etiology}

Much remains unknown about the etiology of SPD. Since it is difficult to study developmental precursors or investigate neurobiological substrates in human studies, an additional avenue of research is the pursuit for plausible and valid animal models of SPD. In addition, genetic and familial studies provide a window into etiologic factors contributing to the expression of SPD symptoms.

Prenatal environmental factors. The Harlow Primate Lab in Madison Wisconsin investigates issues relevant to SPD-SOR in non-human primates. A tactile SP Scale for Monkeys, based on the Sensory Challenge Protocol test paradigm for humans (Miller et al., 2001) was developed and implemented. This test paradigm reliably grouped non-human primates into normal and SPD-SOR, in the tactile domain (Schneider et al., 2007). Specifically, adult non-human primates that had been exposed to prenatal alcohol or stress were identified as SPD-SOR compared to monkeys from normal pregnancies. These SPD-SOR primates also demonstrated significantly lower muscle tone and righting reactions at birth, exhibited significantly greater cortisol levels upon maternal separation at age 6 months, and had impaired cognitive performance on a learning task at 32 months. Schneider et al. (2007) hypothesized and tested whether sensory over-responsiveness is associated with altered dopamine function in the striatum, a critical regulator of frontal-striatal function and a circuit involved in inhibitory control. They hypothesized that poor inhibitory control and/or difficulty filtering information was related to increased responsiveness to sensory stimulation. Increased striatal dopamine type 2 (D2) receptor binding was associated with increased aversive responses to repetitive tactile stimuli and reduced habituation across trials (Schneider et al., 2007, 2008). These studies establish that a SPD-SOR phenotype can be identified in non-human primates, suggest that prenatal stressors may increase vulnerability to SPD, and suggest that sensory over-responsiveness may be related to up-regulation or super-sensitivity of striatal D2 receptors.

Genetic and familial associations. Ayres hypothesized that a genetic vulnerability in some children may contribute to SPD. Recent findings in twin studies indicate that auditory and tactile over-responsivity occur significantly more often in identical than in fraternal twins (e.g., concordance rates of tactile over-responsivity in identical twins was 0.82 compared to 0.27 in fraternal twins; Goldsmith et al., 2006). These findings suggest that the SPD-SOR subtype may have a genetic link. Notably, approximately $45 \%$ of children who screened positive for SPD had no other DSM diagnoses (H.H. Goldsmith, personal communication), a finding which is now cross-validated (A. Carter, personal communication).

\section{Signs and symptoms}

Research on the signs and symptoms of SPD is endeavoring to develop an objective method to identify and differentiate subtypes of SPD. Currently, identification of SPD is based on the global clinical impression of experts, who use information from observation or from screening tools that rely on parent or teacher report-measures such as the Sensory Profile (Dunn and Chadwick, 1999) and the Sensory Processing Measure (Parham and Ecker, 2007). Recent study has been directed toward developing The Sensory Processing Scales (Schoen et al., 2008), which has items in each of the proposed six subtypes of SPD and includes both a direct performance measure (administered directly to the child) and a companion parent/selfrated checklist. A research edition of the SP Scales has preliminary evidence of reliability and validity $(n=200$; Schoen et al., 2008).

\section{Prevalence}

In a retrospective study of a national randomly sampled group of typically developing children (ages $2.5-19 ; n=2410$ ), the bottom $5 \%$ of children with ADHD symptoms and the bottom $5 \%$ of children with SP symptoms were selected for further study $(n=181)$. Of the entire group that were positive for symptoms of either ADHD or SPD-SOR, $40 \%$ had symptoms of both, and about $30 \%$ had symptoms of only ADHD or only SPD-SOR (Ahn et al., 2004). These data suggest that SPD-SOR may be a separate syndrome from ADHD. Clearly, discrimination of SPD from other neurodevelopmental disorders is an important area for future studies. In another study, 1796 families were sampled during a kindergarten screening regarding the presence of abnormal sensory symptoms in their child. Of the total population of the school district, 710 (39\%) returned the survey. Using a conservative estimate that all non-returned surveys 
were negative for SPD-SOR, the rate of significant sensory issues (defined as lower than $3 \mathrm{SD}$ from the mean of the norm reference group for the scale) was 5\% (Ahn et al., 2004).

Epidemiologic data are provided in a new population-based prospective study of a New Haven birth cohort followed for 7 years ( $n=1788$ ). Findings estimate that $16 \%$ of the general population have symptoms of SPD-SOR (Ben-Sasson et al., 2009). Children with higher levels of SOR symptoms at ages $2-4$ had higher reported impairments at age 7 with higher frequencies of internalizing, externalizing and dysregulation problems as well as more problems in social adaptive behavior (Ben-Sasson et al., 2009).

\section{Underlying neural features}

Current research on the underlying neurobiology of SPD has been guided by the overarching hypothesis that both autonomic and central nervous system processes are affected in SPD. These hypotheses are based on Ayres' proposal that children with SPD may have an impaired ability to integrate stimuli from multiple sensory modalities, leading to atypical behavioral responses to ordinary environmental stimuli (Ayres, 1963). Those with SPD-SOR are easily overwhelmed by daily sensory experiences, while those with SPD-SUR tend to "tune-out" or not notice the same sensory experiences (Miller et al., 2007b).

Autonomic nervous system research. The first line of this phase of research, which started in 1995, examined physiologic mechanisms of autonomic nervous system regulation in response to sensory stimulation in children with SPD-SOR. This research was based on Ayres' original theoretical constructs, as well as current clinical observations that many children with SPD-SOR exhibit "fight or flight" responses to everyday stimuli (Ayres, 1963). Atypical sympathetic and parasympathetic nervous system function were documented in children with SPD-SOR compared to typically developing controls (McIntosh et al., 1999; Miller et al., 2001; Schaaf et al., 2003). The sympathetic (increased reactivity) and parasympathetic responses (less vagal variability) were hypothesized to contribute to the inability of children with SPD-SOR to modulate the degree, intensity, and type of response to typical environmental sensory stimuli. When further investigated in relation to other established diagnostic categories, children with SPD-SOR exhibited different physiological patterns than children with attention deficit/hyperactivity disorder (ADHD; Mangeot et al., 2001) as well as children with autism spectrum disorder (ASD; S.A. Schoen et al., submitted). These discriminant validity studies comparing SPD to ADHD and comparing SPD to ASD used both phasic and tonic electrodermal activity as outcome measures. Results of these studies suggest that children with SPD-SOR have more phasic reactivity to sensory stimulation than children with ASD or typically developing children, In addition, children with ASD have lower tonic arousal levels than both typically developing children and children with SPD (S.A. Schoen et al., submitted). On the other hand, children with ADHD displayed atypically large reactions to the initial presentation of a sensory stimulus, but habituated following subsequent presentations of the stimuli (Mangeot et al., 2001; Miller et al., 2001). This finding differentiated children with ADHD from children with SPD-SOR who had larger reactions to all stimuli and did not habituate to recurring sensory stimulation (McIntosh et al., 1999).
Central nervous system research. Studies of the central nervous system (CNS) were then implemented to examine potential brain processes that may underlie SPD. These studies were predicated on Ayres' theory (Ayres, 1972b, 1979) that behavioral expressions of SPD-SOR, such as frequent meltdowns, withdrawal from others, and severe aggression observed after being touched, might be related to immaturity or malfunction in brain processes (Ayres, 1963). Hypotheses were developed for the SPD-SOR and SPD-SUR subtypes, since these children clinically have difficulty integrating multisensory stimuli, or filtering background stimuli. It was hypothesized that auditory stimulation would result in atypical brain activity and that sensory gating and MSI would be abnormal in theses subtypes. Hypotheses were studied in both children (Davies and Gavin, 2007; Brett-Green et al., submitted) and adults (Kisley et al., 2004).

Sensory gating, a measure of the ability of the CNS to inhibit responses to redundant or irrelevant sensory stimuli, manifests electrophysiologically as a reduction in amplitude of a specific evoked potential component (e.g., P50) in response to paired stimuli. When typically developing children were presented with tones at different frequencies and intensities, distinct brain responses were elicited for each intensity (Davies et al., 2009). However, children with SPD-SOR and SPD-SUR did not exhibit the typical increased response to an increased intensity of stimulation, strongly suggesting that their brain processing of simple auditory stimuli is less organized (Davies et al., 2009). The clinical sample also demonstrated less auditory sensory gating than typically developing children (Davies and Gavin, 2007; Davies et al., 2009), suggesting that children with SPD have more difficulty filtering out repeated or irrelevant sensory information. While sensory gating improved with age for typically developing children, sensory gating did not improve with age in the clinical group, indicating that the maturational course of sensory gating in SPD may be quite different than typical controls (Davies and Gavin, 2007; Davies et al., 2009). The brain activity measured in the intensity and gating paradigms correctly classified children with SPD vs. typically developing children with $86 \%$ accuracy (Davies and Gavin, 2007). Moreover, findings suggested that children with SPD could be either over-responsive or under-responsive when responding to sensory input compared to typically developing children (Davies and Gavin, 2007). These studies support Ayres' theories suggesting that the CNS has a deficit in processing sensory stimuli and validates the SOR and SUR subtypes in the current clinical nosology groupings (Miller et al., 2007c).

Sensory gating was also investigated in adults who endorsed high rates of SOR. Adults with greater SPD-SOR symptoms exhibited less efficient sensory gating (i.e., lower suppression of auditory ERP P50 and N100 components in a paired click paradigm; Kisley and Cornwell, (2006)). Notably, decreased P50 responses were correlated with higher endorsement of "perceptual modulation" difficulties (feelings of being flooded by sounds), whereas decreased N100 responses were correlated with increased endorsement of an inability to filter out background sounds. These data suggest that two distinct neural processes might account for these two distinctive types of abnormal auditory information processing.

Additionally, sensory gating was evaluated in rats using prepulse inhibition (PPI), a functional measure of sensorimotor gating that provides a way to investigate how the brain normally suppresses 
over-reactivity to sensory stimuli. SP deficits and PPI deficits in particular were hypothesized to involve disrupted nicotinic acetylcholine signaling in the CNS (see for review Heath and Picciotto, 2009). PPI was pharmacologically impaired and unimodal (auditory startle with auditory prepulse) and bimodal (tactile startle with auditory prepulse) PPI responses to clozapine and nicotine were evaluated. Bimodal PPI impairments in sensory gating were attenuated only by clozapine (Levin et al., 2007), but unimodal PPI impairments in sensory gating were attenuated by a combination of nicotine with clozapine (Levin et al., 2005). While the basic neurotransmitter mechanism of each of these pharmacological agents in isolation is relatively well known, the specific mechanisms of their interactions in modifying unimodal and biomodal PPI needs further study. A better understanding of the basic neurobiology, neurotransmitter interactions, and pharmacology of unimodal versus bimodal PPI may lead to insights about the underlying mechanisms of SPD and perhaps future pharmacological interventions.

Studying MSI in children with SPD was also a logical line of research to pursue given Ayres' fundamental premise that SPD reflects a disorder in "intersensory/MSI." Additionally, clinical observations report children identified with SPD-SOR are overwhelmed in situations where sensations from multiple modalities are present (e.g., a busy mall or a school cafeteria). Below is a brief overview of some preliminary findings that provide a basis for the ongoing research studies of MSI in individuals with SPD.

The ability of the brain to integrate sensory stimuli is a fundamental neural function that must be intact for a person to function adaptively in the environment by responding to meaningful stimuli and ignoring stimuli that are incidental. Recent research based on electroencephalography using event-related potentials (ERPs) suggests that impaired MSI in children with SPD-SOR may be based on differences in the spatio-temporal pattern of MSI responses in SPD compared to typically developing children (B. Brett-Green, personal communication). In a preliminary study of typically developing children auditory-somatosensory MSI was identified based on differences between multisensory and summed unisensory responses (Brett-Green et al., 2008), a classic approach commonly used in MSI research (Stein and Meredith, 1993). MSI was found contralateral to the side of somatosensory stimulation over the caudo-medial auditory cortex in typically developing children (Brett-Green et al., 2008) whereas in children with SPD, frontal regions were activated (B. Brett-Green, submitted). The location of MSI in typically developing children is consistent with recent MSI reports in typical adults (Foxe and Schroeder, 2005; Murray et al., 2005).

In typically developing children, MSI was found in the ipsilateral cortex and in midline cortical regions (Brett-Green et al., 2008). Children with SPD-SOR showed smaller amplitude MSI responses in midline and frontal cortical regions, and showed an absence of ipsilateral integration (B. Brett-Green, submitted). These studies suggest that the automatic integration of multisensory stimuli occurring early in sensory information processing in lower level cortical regions may not occur in children with SPD-SOR. Instead, higher-level frontal MSI processes may be engaged. The possible ramification of this finding may explain why children with SPD-SOR tend to attend to all environmental stimuli, meaningful or not. However, the exact consequence of the differences between typically developing children and those with SPD-SOR or SPD-SUR in MSI is not yet known. The lack of ipsilateral MSI in children with SPD-SOR needs further study.

The evidence that abnormalities in MSI exist in SPD provided the impetus for this special journal issue of Frontiers in Integrative Neuroscience. The comingling of ideas from basic science and from the clinical perspective will benefit research in both fields.

\section{RESEARCH RELATED TO THE INTERVENTION}

Another area that provides evidence of syndrome validity is differential effectiveness of treatment. Although occupational therapists have been treating individuals with SPD for nearly half a century, early studies examining the effectiveness of occupational therapy with a sensory integration approach were fraught with methodological problems (Bailey, 1978; Jenkins et al., 1983; Huff and Harris, 1987). A rigorous randomized clinical trial comparing occupational therapy with a sensory integration approach to an active placebo and a passive placebo was recently conducted (Miller et al., 2007b). The findings suggest that the sensory integration approach is significantly more effective in remediating functional difficulties identified by parents in children with SPD-SOR as well as in improving social/cognitive deficits. Studies measuring the differential treatment effectiveness with various clinical conditions are yet to be completed.

\section{NEUROSCIENCE RELATED TO SENSORY INTEGRATION EARLY NEUROSCIENCE RESEARCH}

Historically, neuroscience research examined the function of individual sensory modalities (audition, vision, olfaction, gestation, touch) in isolation. Sensory modalities were often further explored by submodalities. For example, vision research focused on color, form, shape, orientation, and depth perception. While studies examining single sensory modalities have informed our understanding of SP, neuroscientists realize that real world experiences are multisensory in nature, and thus studying how the senses interact is essential. Ayres shared this viewpoint and frequently wrote about how crucial multisensory experiences were for normal development and how those with SPD had deficits in integrating input from multiple sensory modalities (Ayres, 1958, 1961). Further, she felt that a lack of integrated sensory perceptions in SPD related to their atypical behaviors (Ayres, 1972b, 1979). In neuroscience, interest in multisensory processing and increased technological advances have led to numerous studies showing that interactions among the senses profoundly influence behavior, perception, emotion and cognition (see for review Welch and Warren, 1986; Stein and Meredith, 1993; Calvert et al., 2004). Currently, a major focus of neuroscience research, from the cellular to whole organism level, is focused on examining how multiple sensory systems interact.

Illusions were examined in classic experiments that guided the neuroscience field toward the modern study of MSI. Illusions provide some of the most overt indications that senses interact. The most renowned example of illusion research is the ventriloquism effect, in which simultaneous auditory and visual information emanate from different locations in space (Howard and Templeton, 1966). Most people perceive the ventriloquist's voice coming from the dummy rather than the ventriloquist; in other words, the visual system "captures" the auditory information. Another classic illusion, 
the "McGurk effect" (McGurk and McDonald, 1976), occurs when the sights and sounds associated with speech are mismatched. This illusionary effect involves showing a video of a person saying one phoneme, e.g., "ba", while the sound being played is another phoneme, e.g., "ga". The perception of those watching and listening is a third phoneme such as "da". These examples and many more provide insight into how the brain processes sensory input, what the natural limitations are, and how "misperceptions" can exist. Current understanding of how the senses interact is based in part on these and other early MSI studies.

\section{BEHAVIORAL CONSEQUENCES OF MSI}

Experiencing the environment as a coherent whole requires constant combining and interpreting multiple elements of sensory information. The brain processes and interprets abundant information received from environmental stimuli in order to determine which stimuli are irrelevant vs. which stimuli are relevant and require a behavioral response. Behaviors crucial for basic survival, including enhanced detection, localization, and orienting to pertinent environmental stimuli are all consequences of appropriate MSI (Stein et al., 1988; Stein and Meredith, 1993). For example, classic experiments demonstrate that perception is better and reaction times are faster to multisensory stimuli that occur in the same location and time compared to unisensory stimuli (Welch and Warren, 1986; Calvert et al., 2004). A comprehensive review of early research examining intersensory interactions and behavior is beyond the scope of this article, but is provided by Welch and Warren (Welch and Warren, 1986).

\section{CONSTRUCTS RELATED TO MSI MSI in the superior colliculus}

Stein, Meredith, and Wallace (Meredith and Stein, 1986; Meredith et al., 1987; Stein and Meredith, 1993) are widely credited with the initiation of modern MSI research. They developed the preeminent model for researching MSI in the superior colliculus (SC) of cats. Among their contributions was the identification of three neurophysiologic principles of MSI: the spatial, temporal, and inverse effectiveness principles. The spatial principle states that multisensory stimuli that are coincident in space result in enhanced SC neuronal responses, but stimuli that are separated in space result in standard or depressed SC neuronal responses (Meredith and Stein, 1986). This spatial principal is due, in part, to overlapping topologies of the receptive fields in the SC (Stein and Meredith, 1993). The temporal principle states that multisensory stimuli that are coincident in time result in enhanced SC neuronal responses, but stimuli that are separated in time result in standard or depressed SC neuronal responses (Meredith et al., 1987). An optimal temporal window exists in which MSI can occur (Meredith et al., 1987). Furthermore, when multisensory stimuli are coincident in both space and time, SC neuronal responses can be superadditive (i.e., greater than the sum of the responses to the unisensory stimuli; Meredith and Stein, 1983). The principle of inverse effectiveness states that when the unisensory components of a compound stimulus, experienced in isolation, evoke relatively weak responses, the composite multisensory stimuli result in enhanced SC neuronal responses; specifically, the effectiveness of the individual sensory components are amplified when combined, conferring greater importance to the multisensory stimuli (Meredith and Stein, 1983). Subsequent experiments demonstrated that these principles governing MSI in neurons also influence behavioral responses (e.g., detection is improved and orienting is faster and more accurate for multisensory compared to unisensory stimulation; Stein et al., 1988). These latter studies established an important link between neuronal function and behavior.

\section{MSI in the cortex}

MSI occurs in multiple areas. In addition to subcortical regions, such as the SC and thalamus, many cortical regions have been implicated in MSI, including the parietal cortex, superior temporal cortex, and frontal cortex (Calvert et al., 2004). A basic pre-requisite for MSI is anatomical convergence of input from the different sensory systems on individual neurons or groups of neurons (Adrian, 1949; Stein and Meredith, 1993). Originally, cortical MSI was attributed only to association regions where convergence of input from different sensory systems had been demonstrated. However, more recent research demonstrates that MSI occurs in regions previously considered to be unisensory (Foxe et al., 2000; Macaluso, 2006). In fact, some neuroscientists suggest that the entire neocortex is essentially multisensory (Ghazanfar and Schroeder, 2006). MSI appears to be a ubiquitous brain function, occurring in many different areas and during different stages of sensory information processing.

Various research endeavors are investigating whether the basic principles governing MSI in the SC are the same in the cortex and at the human behavioral level. While the three basic MSI principles appear to be applicable to cortical and behavioral responses to multisensory stimuli, evolving knowledge suggests that cortical integration of multisensory stimuli and especially human behavioral responses to multisensory stimuli are more complex than the neuronal responses observed in the SC (Murray et al., 2005; Ross et al., 2007; Bizley and King, 2008). Of the many issues related to the properties of MSI in the cortex, and how MSI influences behavior, three are highlighted below: timing of cortical MSI, how attention effects MSI, and sensory dominance.

Timing of MSI. One area of research investigates at which stage of sensory information processing the brain utilizes MSI to guide behavior. Recent indications are that cortical MSI occurs very early during SP, in fact earlier than previously thought, challenging the assumption that cortical integration is one of the last phases in the hierarchy of sensory information processing (Foxe and Simpson, 2002; Molholm et al., 2002; Foxe and Schroeder, 2005; Macaluso, 2006). Data suggest that early cortical use of multisensory information may immediately influence behavior, possibly prior to conscious perception of the stimuli (Foxe and Schroeder, 2005). As Ayres advocated in her intervention model, utilizing planned and controlled sensory inputs without directing conscious attention to the stimuli can be very powerful in changing behavior (Ayres, 1972b).

Attention influences MSI. Controlling responses to objects and events in the environment requires selection of information, which can be modified by attention. Attention facilitates behavioral responses to stimuli that are important (Eimer and Driver, 2001). Attention has been demonstrated to consciously (e.g., with attended stimuli) and unconsciously (e.g., with unattended stimuli) influence 
MSI, such that cortical ERPs are generally larger for attended stimuli than for unattended stimuli (Eimer and Driver, 2001; Hötting et al., 2003; Lloyd et al., 2003; Talsma and Woldorff, 2005). Thus, MSI can be influenced at both pre-perceptual and post-perceptual stages of sensory information processing. An overarching concept is that MSI is a dynamic and complex process involving the coordinated activation and possible parallel organization of numerous cortical and subcortical brain regions at multiple stages of sensory information processing.

\section{Sensory dominance}

Recent studies suggest that sensory dominance is multifaceted at the behavioral level. Sensory dominance has been studied in both adults and infants as young as 4 months of age (Lewkowicz, 2000, 2003). While spatial and temporal aspects of sensory stimuli are extremely important, sensory dominance does not exclusively depend on whether the sensory information is spatial or temporal in nature, rather they are adaptable to specific aspects of the task or context as well as previous sensory experiences of the individual (Fort et al., 2002; Molholm and Foxe, 2004; Murray et al., 2005; Teder-Sälejärvi et al., 2005; Navarra et al., 2007; Ross et al., 2007). In some situations dominance is a function of which of the sensory modalities is most accurate in detecting the sensory information. This type of dominance has been referred to as modality appropriate dominance. For example, in adults the visual system has been shown to be more precise in estimating/perceiving direction (Westheimer and McKee, 1977) and is often dominant in spatial tasks, while the auditory system is considered dominant in temporal tasks (Welch and Warren, 1980). Generally, adult humans are visually dominant, however, in certain situations such as when the visual image is not optimal, audition dominates (Alais and Burr, 2004). Thus, perception and behavior are affected by properties of the individual sensory systems as well as multisensory stimulus properties. Furthermore, recent studies suggest that MSI is statisticallyoptimal (Ernst and Banks, 2002; Battaglia et al., 2003; Alais and Burr, 2004; Knill and Pouget, 2004) and that behavioral patterns of animals and humans follows Bayesian-optimal strategies (Deneve and Pouget, 2004; Lalanne and Lorenceau, 2004; Montagnini et al., 2007; Rowland et al., 2007; Ma et al., 2008). Collectively, research demonstrates that the CNS is flexible and accommodates to the needs of the individual due to the constantly and rapidly changing environment. Similarly, Ayres (1972b) noted that a persistent and problematic symptom of children with SPD is impairment in automatically adapting to rapidly changing sensory environments.

\section{MSI Developmental trajectory}

Studies examining the relationship of the development of multisensory neurons in the SC compared to cortical function found multisensory neurons in the SC are immature and unable to integrate multisensory input they receive at first (Wallace et al., 1992). However, maturation increases the neuron's ability to integrate multisensory input, but only if functional input is received from the cortex (Wallace and Stein, 2007). The maturation process is thought to be developmentally gated and guided by postnatal experience (Wallace, 2004). Developmental studies in cats, non-human primates, and humans have demonstrated that multisensory circuits and their integrative capabilities are established over an extended postnatal period (for review see Wallace, 2004; Wallace et al., 2004; Stein et al., 2009). Early multisensory neurons do not exhibit response enhancement or depression, as seen in adult neurons, regardless of the spatial or temporal or physical features of the compound stimuli; instead all responses appear as if they are unimodal responses (Wallace, 2004; Wallace et al., 2004). Additionally, the temporal window for MSI becomes increasingly refined during development, with the largest window observed in infancy (Lewkowicz and Kraebel, 2004). Thus, the developmental trajectory of MSI depends on many factors a few of which are highlighted below.

MSI developmental in normal environments. The maturational processes of multisensory neurons are extremely sensitive to multimodal experiences and interactions with the environment during development. In fact, human infants do not appear to have an established sensory dominance hierarchy, instead they are more dependent on the type of sensory information available (Lewkowicz, 2000, 2003). In infants, when speech sounds are adult-like infants are visually dominant, but speech that is more rhythmic or singsong results in auditory dominance (Lewkowicz, 2000, 2003). Recent studies suggest that optimal integration is learned from experience and interaction with the environment and may not be optimal before 8 years of age (for review see Ernst, 2008). Similarly, current clinical experience shows that early intervention is likely to be most effective for children with clinical disorders (Spittle et al., 2007; Howlin et al., 2009; Nair et al., 2009).

MSI development in altered environments. Experiences and interaction with the environment during development have an enormous impact on adult functional behavior. Illustrations of how environment and development interact to alter functional behavior are provided by studies that manipulate the environment during development. Environmental manipulations include changing the normal spatial or temporal relationship of multimodal stimuli and sensory deprivation. For example, artificially constraining the environment during development so that multimodal stimuli are always spatially disparate prevents the development of normal multisensory enhancement to spatially coincident stimuli and results in enhancement only to stimulus in mismatched combinations that were experienced during development (for review see Wallace and Stein, 2007). Additionally, elimination of all visual experience from birth to adulthood, in animals, effects not only unisensory (visual) processing, but also the ability to integrate multisensory cues from other sensory modalities (Wallace et al., 2004). Behavior of adult animals that have been deprived of normal sensory experiences resembles behavior observed in normally reared animals at earlier developmental ages. Similarly, Ayres noted that children with SPD often display behavior that is reflective of an earlier stage of development (Ayres, 1961; Walker, 1993).

\section{MSI plasticity}

Evidence suggests that interactions with the environment change MSI and that plasticity may continue throughout the lifespan. For example, when individuals wear optical lenses that invert the visual image, after a period of time the image is perceived as right side up (Kohler, 1962). Studies also demonstrate that even though 
unisensory deficits are apparent in older adults, presentation of multisensory stimuli can improve response time to specific tasks (Laurienti et al., 2006). Generating a perception of one's body in external space is essential for effective interaction with the environment. To generate a representation of the body, the brain utilizes multiple spatial references (Maravita et al., 2003), which relate to both the space around the body and the space beyond. MSI is dynamic and automatically and constantly changes as the individual interacts with the environment (Holmes and Spence, 2004). For example, the use of a tool (e.g., a hammer) extends one's personal space. These studies demonstrate that the brain continuously utilizes environmental information in a contextually coordinated and adaptable manner. Ayres conceptualized SPD as a disorder of body scheme in which children misperceive their immediate space and their surrounding space (Ayres, 1961). She believed that therapy based on a "sensory integration approach" would normalize the spatial perceptions from multiple sensory systems (Ayres, 1975) and contribute to successful participation in daily life activities.

\section{TRANSLATIONAL RESEARCH}

From this brief review of the clinical disorder, the widespread use of sensory integration treatment, and the overview of related neuroscience, it is apparent that there is significant potential for translational research. Crucial to moving research on SPD forward is the clarification that abnormal MSI is only one of many possible underlying causes. Evaluation of MSI was a logical avenue to pursue given Ayres' original theory. However, current lack of data on other potential impairment in SPD does not imply that none exist. Three categories where translational research could expand the study of SPD are highlighted: differential diagnosis, intervention, and theory development.

\section{DIFFERENTIAL DIAGNOSIS OF SPD}

Development of an objective method to diagnosis SPD and to differentiate it from other neurodevelopmental and neurobehavioral conditions is essential. New syndromes are generally defined based on a framework differentiating signs and symptoms, neurological features, etiologies, and developmental course. An improved diagnostic method is needed and would be particularly useful if it included objective laboratory measures to differentiate SPD from other childhood conditions. Identification of biological markers, such as a specific pattern of MSI in children with SPD compared to typically developing children and children with other clinical diagnoses, would contribute greatly to improving differential diagnosis of SPD.

Comorbidity is one of the most challenging issues in studies of childhood developmental and behavioral disorders (Kendell and Jablensky, 2003). Since there is no current overarching theory regarding the specific impairment underlying the behavioral manifestations of SPD, current hypotheses have been based on Ayres' original theories and recent clinical observations of children with SPD. The combination of constructs based on the original literature from Ayres (1972b) and current clinical observations contributed to development of the studies discussed earlier, which were designed to shed light on the question/issue of differences between children with SPD and typically developing children. However, a theoretical model specifying the possible neurological impairments in SPD would further define and prioritize studies. This could ultimately lead to more effective differentiation of children with SPD from other clinical groups.

Most developmental and behavioral diagnoses in childhood are obtained using criteria in either the DSM-IV (American Psychiatric Association, 1994) or the International Classification of Diseases (ICD)-9 (World Health Organization, 2004). The DSM and ICD are comprised of lists of behavioral features designating specific diagnoses. However, often a final diagnosis is made based on the Global Clinical Impression, which ultimately is a subjective judgment (American Psychiatric Association, 1994; Jensen et al., 2006). For some disorders, such as ADHD, behavioral scales such as parent and/or teacher ratings, have been developed and standardized. While helpful, these scales are based on personal perceptions (Rogers et al., 2003), a useful viewpoint, but sometimes an unreliable way to define homogeneous groups. A few performance measures are available for identifying SPD, such as the Sensory Integration and Praxis Test (Ayres, 1989), which is age limited and focuses primarily on dyspraxia, and the SP Scales (Schoen et al., 2008), which is in a non-standardized research edition. The development and standardization of a reliable and valid scale to assess SPD is essential for the field to move forward.

An appeal for the rapid advancement in understanding MSI has been made based on the probability that some neurological disorders involve failure of normal MSI (Molholm and Foxe, 2004). Therefore, studying the neurophysiology of MSI in typically developing children and children with SPD is a logical and important line of research to pursue. Compared to the number of MSI studies in typically developing individuals, few MSI studies have been conducted with clinical populations. One study showed that adults with dyslexia integrate auditory and visual information over an extended temporal window, which may reflect a problem processing the visual and auditory (phonemic) components of written words and the participant's difficulty reading (Hairston et al., 2005). Quantitative laboratory paradigms, including but not limited to those that measure MSI, may differentiate SPD as well as other developmental and behavioral disorders.

\section{INTERVENTION}

A variety of neuroscience research supports the use of multisensory strategies to improve behavior. While most studies have focused on learning within a single modality, recent studies examine the effect that intersensory interactions can have on perceptual learning and cognition. For example, compared to a visually trained group, an audio-visual trained group exhibited greater discrimination and detection learning, both within the first session and across multiple training sessions (Kim et al., 2008). Multisensory stimulation has also been found to improve cognitive functioning in mildly impaired Alzheimer's patients (Ozdemir and Akdemir, 2009). In addition, research demonstrates that the use of MSI systems may facilitate recovery from unisensory and spatial deficits. For example, in patients' with visual impairment, the association of a sound with a visual stimulus presented to their neglected field increased perception of the visual stimulus (Frassinetti et al., 2002). These studies further support Ayres' hypothesis regarding the potential for treatment using multiple sensory modalities to ameliorate learning and other developmental difficulties in children with SPD (Ayres, 1958; Walker, 1993). 


\section{THEORY DEVELOPMENT}

Apparent from this brief review is that many of the concepts underlying Ayres' theory, diagnosis and treatment of SPD are still valid. Progress has been made towards developing a theoretical framework within which to move research forward. Increased translational collaboration will contribute greatly towards future efforts.

The time is right for researchers in basic science and clinical researchers to work collaboratively. MSI studies establish an important link between neuronal function and real life behaviors. Important advances have been made defining typical neural mechanisms of MSI in animals and adults and a start has been made with children (Brett-Green et al., 2008). However, understanding how neural mechanisms of MSI are impaired in SPD and the relationship of this problem to other developmental and behavioral disorders needs further study. Although, many of the findings from current MSI research validate Ayres' original hypotheses, refinement and growth of the conceptual model of the disorder and treatment is needed to generate specific hypotheses. For example, the contributions of unisensory versus multisensory impairments, sensory threshold issues, critical age research, and identification of atypicality in brain structures and functions must be included in future studies directed towards theory development. However, the priority of research depends on development of a solid theoretical model, which attempts to relate the phenomenology of the disorder to the neural pathology of the disorder.

\section{REFERENCES}

Adrian, E. D. (1949). The Sherrington Lectures. I: Sensory Integration. Liverpool, University of Liverpool Press.

Ahn, R. R., Miller, L. J., Milberger, S., and McIntosh, D. N. (2004). Prevalence of parents' perceptions of sensory processing disorders among kindergarten children. Am. J. Occup. Ther. 58, 287-302.

Alais, D., and Burr, D. (2004). The ventriloquist effects result from near-optimal bimodal integration. Curr. Biol. 14, 257-262.

American Psychiatric Association (1994). Diagnostic and Statistical Manual of Mental Disorders, DSM-IV edn. Washington, DC,American Psychiatric Association.

Ames, L. B., and Ilg, F. L. (1964). The developmental point of view with special reference to the principle of reciprocal neuromotor interweaving. J. Genet. Psychol. 105, 195-209.

Arendt, R. E., MacLean, W. E., and Baumeister, A. A. (1988). Critique of sensory integration therapy and its application in mental retardation. Am. J. Ment. Retard. 92, 401-411.

Ayres, A. J. (1954). Ontogenetic principles in the development of arm and hand functions. Am. J. Occup. Ther. 8, 95-99, 121.
Ayres, A. J. (1958). The visual-motor function. Am. J. Occup. Ther. 12, 130-138, 155.

Ayres, A. J. (1961). Development of the body scheme in children. Am. J. Occup. Ther. 15, 99-102, 128.

Ayres, A. J. (1963). The development of perceptual-motor abilities: a theoretical basis for treatment of dysfunction. Am. J. Occup. Ther. 27, 221-225.

Ayres, A. J. (1965). Patterns of perceptualmotor dysfunction in children: a factor analytic study. Percept. Mot. Skills 20, 335-368.

Ayres, A. J. (1966a). Interrelation of perception, function, and treatment. J. Am. Phys. Ther. Assoc. 46, 741-744.

Ayres, A. J. (1966b). Interrelations among perceptual-motor abilities in a group of normal children. Am. J. Occup. Ther. 20, 288-292.

Ayres, A. J. (1972a). Overview. In Sensory Integration and Learning Disorders, A. J. Ayres, ed. (Los Angeles, CA, Western Psychological Services), pp. 1-12.

Ayres, A. J. (1972b). Sensory Integration and Learning Disorders. Los Angeles, CA, Western Psychological Services.

Ayres, A. J. (1972c). Some general principles of brain function. In Sensory Integration and Learning Disorders,A. J.Ayres, ed. (Los Angeles,

\section{CONCLUSION}

In 2009, the International Multisensory Research Forum held its 10th conference, 15 years after the seminal work of Stein and Meredith. In 2010, the SPD-SWG will hold its fifth meeting. Despite the growth of the field, application of neuroscience and other scientific findings to clinical practice is challenging. The question is not whether scientists and clinicians should work together, but how to facilitate their collaboration. If scientists and clinicians who are interested in sensory integration began active collaborative studies, their findings could inform one another. A translational approach has the potential to have a profound effect on individuals with SPD and their families as well as increasing knowledge related to neuroscience of MSI.

\section{ACKNOWLEDGMENTS}

The authors wish to first acknowledge the time and energy of the many children and families participating in the studies described here. In addition, the Wallace Research Foundation has provided generous support to all the members of the SPD Scientific Work Group over the past 15 years in the quest for knowledge about Sensory Processing Disorder. The Children's Hospital Research Institute and The General Clinical Research Centers Program at the Children's Hospital of Denver provided additional funding. Special thanks to research assistants, Anna Legenkaya and Jennifer Jones, and to the entire staff at the Sensory Processing Disorder (SPD) Foundation and the Sensory Therapies and Research Center in Greenwood Village, CO.

CA, Western Psychological Services), pp. 13-24.

Ayres, A. J. (1975). Sensorimotor foundations of academic ability. In Perceptual and Learning Disabilities in Children, W. M. Cruickshank and D. P. Hallahan, eds (Syracuse, NY, Syracuse University Press), pp. 301-358.

Ayres, A. J. (1979). Sensory Integration and the Child. Los Angeles, CA Western Psychological Services.

Ayres, A. J. (1989). Sensory Integration and Praxis Tests. Los Angeles, CA, Western Psychological Services.

Ayres, A. J., and Tickle, L. S. (1980). Hyper-responsivity to touch and vestibular stimuli as a predictor of positive response to sensory integration procedures by autistic children. Am J. Occup. Ther. 34, 375-381.

Bailey, D. M. (1978). The effects of vestibular stimulation on verbalization in chronic schizophrenics. Am. J. Occup. Ther. 32, 445-450.

Battaglia, P. W., Jacobs, R. A., and Aslin, R. N. (2003). Bayesian integration of visual and auditory signals for spatial localization. J. Opt. Soc. Am. A, Opt. Image. Sci. Vis. 20, 1391-1397.

Ben-Sasson, A., Carter, A. S., and BriggsGowan, M. J. (2009). Sensory overresponsivity in elementary school: prevalence and social-emotional correlates. J. Abnorm. Child Psychol. [Epub ahead of print].

Bennett, E. L., Diamond, M. C., Krech, D. and Rosenzweig, M. R. (1964). Chemical and anatomical plasticity of brain. Science 146, 610-619.

Bizley, J. K., and King, A. J. (2008). Visual-auditory spatial processing in auditory cortical neurons. Brain Res. 1242, 24-36.

Brett-Green, B., Miller, L. J., Gavin, W. J., and Davies, P. L. (2008). Multisensory integration in children: a preliminary ERP study. Brain Res. 1242, 283-290.

Buser, P., and Imbert, M. (1961). Sensory projections to the motor cortex in cats: a microelectric study. In Principles of Sensory Communication, W. A. Rosenblith, ed. (New York, NY, John Wiley and Sons). pp 606-626.

Calvert, G., Spence, C., and Stein, B. E. (2004).The Handbook of Multisensory Processes. Cambridge, MA, The MIT Press.

Davies, P. L., Chang, W. P., and Gavin, W. J. (2009). Maturation of sensory gating performance in children with and without sensory processing disorders. Int. J. Psychophysiol. 72, 187-197.

Davies, P. L., and Gavin, W. J. (2007). Validating the diagnosis of sensory processing disorders using EEG technology. Am. J. Occup. Ther. 61, 176-189. 
Deneve, S., and Pouget,A. (2004). Bayesian multisensory integration and crossmodal spatial links. J. Physiol. Paris. 98, 249-258.

Denhoff,E. (1981).Current status of infant stimulation or enrichment programs for children with developmental disabilities. Pediatrics 67, 32-37.

Dru, D., Walker, J. P., and Walker, J. B. (1975). Self-produced locomotion restores visual capacity after striate lesion. Science 187, 265-266.

Dunn, C. M., and Chadwick, G. (1999). Protecting Study Volunteers in Research: A Manual for Investigative Sites. Boston, MA, CenterWatch, Inc.

Eimer, M., and Driver, J. (2001). Crossmodal links in endogenous and exogenous spatial attention: evidence from event-related brain potential studies. Neurosci. Biobehav. Rev. 25, 497-511.

Ernst, M. O. (2008). Multisensory integration: a late bloomer. Curr. Biol. 18, R519-R521.

Ernst, M. O., and Banks, M. S. (2002). Humans integrate visual and haptic information in a statistically optimal fashion. Nature 412, 429-433.

Fort, A., Delpuech, C., Pernier, J., and Giard, M. H. (2002). Dynamics of cortico-subcortical cross-modal operations involved in audio-visual object detection in humans. Cereb. Cortex 12, 1031-1039.

Foxe, J. J., Morocz, I. A., Murray, M. M., Higgins, B. A., Javitt, D. C., and Schroeder, C. E. (2000). Multisensory auditory-somatosensory interactions in early cortical processing revealed by high-density electrical mapping. Brain Res. Cogn. Brain Res. 10, 77-83.

Foxe, J. J., and Schroeder, C. E. (2005). The case for feedforward multisensory convergence during early cortical processing. Neuroreport 16, 419-423.

Foxe, J. J., and Simpson, G. V. (2002). Flow of activation from $\mathrm{V} 1$ to frontal cortex in humans. A framework for defining "early" visual processing. Exp. Brain Res. 142, 139-150.

Frassinetti, F., Bolognini, N., and Làdavas, E. (2002). Enhancement of visual percetion by crossmodal visuoauditory interaction. Exp. Brain Res. 147, 332-343.

Ghazanfar, A. A., and Schroeder, C. E. (2006). Is neocortex essentially multisensory? Trends Cogn. Sci. 10, 278-285.

Goldsmith,H.H.,VanHulle,C.A.,Arneson, C. L., Schreiber, J. E., and Gernsbacher, M.A. (2006).A population-based twin study of parentally reported tactile and auditory defensiveness in young children. J. Abnorm. Child Psychol. 34, 393-407.

Green, J. D. (1958). The rhinencephalon and behavior. In Ciba Foundation
Symposium on the Neurological Basis of Behaviour, G. E. W. Wolstenholme and C. M. O'Connor, eds (London, J. \& A. Churchill). pp. 225-235.

Hairston, W. D., Burdette, J. H., Flowers, D. L., Wood, F. B., and Wallace, M. T. (2005). Altered temporal profile of visual-auditory multisensory interactions of dyslexia. Exp. Brain Res. 166, 474-480.

Heath, C. J., and Picciotto, M. R. (2009). Nicotine-induced plasticity during development: modulation of the cholinergic system and long-term consequences for circuits involved in attention and sensory processing. Neuropharmacology 56, 254-262.

Hebb, D. O. (1949). Organization of Behavior. New York, NY, Wiley.

Herrick, C. J. (1956). The Evolution of Human Nature. Austin, TX, University of Texas Press.

Holmes, N. P., and Spence, C. (2004). The body schema and multisensory representation(s) of peripersonal space. Cogn. Process. 5, 94-105.

Hötting, K., Rösler, F., and Röder, B. (2003). Crossmodal and intermodal attention modulate event-related brain potentials to tactile and auditory stimuli. Exp. Brain Res. 148, 26-37.

Howard, I. P., and Templeton, W. B. (1966). Human Spatial Orientation. London, Wiley.

Howlin, P., Magiati, I., and Charman, T. (2009). Systematic review of early intensive behavioral interventions for children with autism. Am. J. Intellect. Dev. Disabil. 114, 23-41.

Huff, D.M., and Harris, S.C. (1987). Using sensorimotor integrative treatment with mentally retarded adults. Am. J. Occup. Ther. 41, 227-231.

Hyden,H., and Egyhazi, E. (1962). Nuclear RNA changes of nerve cells during a learning experiment in rats. Proc. Natl. Acad. Sci. U.S.A. 48, 1366-1373.

Jenkins, J. R., Fewell, R., and Harris, S. R. (1983). Comparison of sensory integrative therapy and motor programming. Am. J. Ment. Defic. 88, 221-224.

Jensen, P. S., Knapp, P., and Mrazek, D. A. (2006). Toward a New Diagnostic System for Child Psychopathology. New York, NY, The Guilford Press.

Jung, R., Kornhuber, H. H., and DaFonseca, J. S. (1963). Multisensory convergence on cortical neurons. In Brain Mechanisms, G. Moruzzi, A. Fessard and J. J. Japser, eds (New York, NY, Elsevier Publishing, Co.). pp. 207-240.

Kendell, R., and Jablensky, A. (2003). Distinguishing between the validity and utility of psychiatric diagnoses. Am. J. Psychiatry 160, 4-12.

Kim, R.S.,Seitz,A.R., and Shams, L. (2008). Benefits of stimulus congruency for multisensory facilitation of visual learning. PLoS ONE 3, e1532. doi 10.1371/journal.pone.0001532

Kisley, M. A., Noecker, T. L., and Guinther, P.M. (2004).Comparison of sensory gating to mismatch negativity and self-reported perceptual phenomena in healthy adults. Psychophysiology 41, 604-612.

Kisley, M.A., and Cornwell, Z.M. (2006). Gamma and beta neural activity evoked during a sensory gating paradigm: effects of auditory, somatosensory and cross-modal stimulation. Clinical Neurophysiology 117, 2549-2563.

Knill, D. C., and Pouget, A. (2004). The Bayesian brain: the role of uncertainty in neural coding and computation. Trends Neurosci. 27, 712-719.

Kohler, I. (1962). Experiments with goggles. Sci. Am. 206, 62-72.

Lalanne, C., and Lorenceau, J. (2004). Crossmodal integration for perception and action. J. Physiol. (Paris) 98, 265-279.

Lassek, A. M. (1957). The Human Brain from Primitive to Modern. Springfield, MA, Charles C Thomas.

Laurienti, P. J., Burdette, J. H., Maldjian, J. A., and Wallace, M. T. (2006). Enhanced multisensory integration in older adults. Neurobiol. Aging 27, 1155-1163.

Levin, E. D., Caldwell, D. P., and Perraut, C. (2007). Clozapine treatment reverses dizocilpine-induced deficits of prepulse inhibition of tactile startle response. Pharmacol. Biochem. Behav. 86, 597-605.

Levin, E. D., Petro, A., and Caldwell, D. P. (2005). Nicotine and clozapine actions on pre-pulse inhibition deficits caused by $N$-methyl-D-aspartate (NMDA) glutamatergic receptor blockade. Prog. Neuropsychopharmacol. Biol. Psychiatry 29, 581-586.

Levin, S., and Alpert, M. (1959) Differential maturation of the central nervous system as a function of early experience. Arch. Gen. Psychiatry 1, 403-405.

Lewkowicz, D. J. (2000). Infants' perception of the audible, visible, and bimodal attributes of multimodal syllables. Child Dev. 71, 1241-1257.

Lewkowicz, D. J. (2003). Learning and discrimination of audiovisual events in human infants: the hierarchical relation between intersensory temporal synchrony and rhythmic pattern cues. Dev. Psychol. 39, 795-804.

Lewkowicz, D. J., and Kraebel, K. S. (2004). The value of multisensory redundancy in the development of intersensory perception. In The Handbook of Multisensory Processes, G. Calvert, C Spence and B. Stein, eds (Cambridge, MA, The MIT Press), pp. 655-678.
Lloyd, D. M., Merat, N., McGlone, F., and Spence, C. (2003). Crossmodal links between audition and touch in covert endogenous spatial attention. Percept. Psychophys. 65, 901-924.

Luria, A. R. (1966). Higher Cortical Functions in Man. New York, NY, Basic Book.

Ma, W. J., Beck, J. M., and Pouget, A. (2008).Spiking networks for Bayesian inference and choice. Curr. Opin. Neurobiol. 18, 217-222.

Macaluso, E. (2006). Multisensory processing in sensory-specific cortical areas. Neuroscientist 12, 327-338.

Mangeot,S.D., Miller,L.J.,McIntosh, D. N., McGrath-Clarke, J., Simon, J., Hagerman, R. J., and Goldson, E. (2001). Sensory modulation dysfunction in children with attentiondeficit-hyperactivity disorder. Dev. Med. Child Neurol. 43, 399-406.

Maravita, A., Spence, C., and Driver, J. (2003). Multisensory integration and the body schema: close to hand and within reach. Curr. Biol. 13, R531-R539.

McGurk, H., and McDonald, J. (1976). Hearing lips and seeing voices. Nature 264, 746-748.

McIntosh, D. N., Miller, L. J., Shyu, V., and Hagerman, R. (1999). Sensorymodulation disruption, electrodermal responses, and functional behaviors. Dev. Med. Child Neurol. 41, 608-615.

Melzack, R. (1962). Effects of early perceptual restriction on simple visual discrimination. Science 137, 978-979.

Meredith, M. A., Nemitz, J. W., and Stein, B. E. (1987). Determinants of multisensory integration in superior colliculus neurons. I. Temporal factors. J. Neurosci. 7, 3215-3229.

Meredith, M. A., and Stein, B. E. (1983). Interactions among converging sensory inputs in the superior colliculus. Science 221, 389-391.

Meredith, M. A., and Stein, B. E. (1986). Visual, auditory, and somatosensory convergence of cells in superior colliculus results in multisensory integration. J. Neurophysiol. 56, 640-662.

Miller, L., Anzalone, M., Lane, S., Cermak, S., and Osten, E. (2007a). Concept evolution in sensory integration: a proposed nosology for diagnosis. Am. J. Occup. Ther. 61, 135-140.

Miller, L. J., Coll, J. R., and Schoen, S. A. (2007b). A randomized controlled pilot study of the effectiveness of occupational therapy for children with sensory modulation disorder. Am. J. Occup. Ther. 61, 228-238.

Miller, L. J., Anzalone, M. E., Lane,S. J., Cermak, S. A., and Osten, E. T. (2007c). Concept evolution in sensory integration: a proposed nosology for diagnosis. Am. J. Occup. Ther. 61, 135-140. 
Miller, L. J. (2006). Sensational Kids: Help and Hope for Children with Sensory Processing Disorder. New York, NY, Putnam.

Miller,L. J., Reisman, J.E., McIntosh, D. N., and Simon, J. (2001). An ecological model of sensory modulation: performance of children with fragile X syndrome, autistic disorder, attention-deficit/hyperactivity disorder, and sensory modulation dysfunction. In Understanding the Nature of Sensory Integration with Diverse Populations, S. S. Roley, E. I. Blanche and R. C. Schaaf, eds (San Antonio, TX, Therapy Skill Builders), pp. 57-88.

Molholm, S., and Foxe, J. J. (2004). Look 'hear', primary auditory cortex is active during lip-reading. Neuroreport 16, 123-124.

Molholm, S., Ritter, W., Muttay, M. M., Javitt, D. C., Schroeder, C. E., and Foxe, J. J. (2002). Multisensory auditory-visual interactions during early sensory processing in humans: a high density electrical mapping study. Cogn. Brain Res. 14, 115-128.

Montagnini,A., Mamassian,P.,Perrinet, L., Castet, E., and Masson, G. S. (2007). Bayesian modeling of dynamic motion integration. J. Physiol. (Paris) 101, 64-77.

Murray,M.M.,Molholm,S.,Michel, C. M., Heslenfeld, D. J., Ritter, W., Javitt, D. C., Schroeder, C. E., and Foxe, J. J. (2005). Grabbing your ear: rapid auditorysomatosensory multisensory interactions in low-level sensory cortices are not constrained by spatial alignment. Cereb. Cortex 15, 963-974.

Nair, M. K., Philip, E., Jeyaseelan, L., George, B., Mathews, S., and Padma, K. (2009). Effect of Child Development Centre model early stimulation among at risk babies - a randomized controlled study. Indian Pediatr. 46, s20-s26.

Navarra, J., Soto-Faraco, S., and Spence, C. (2007). Adaptation to audiotactile asynchrony. Neurosci. Lett. 413, 72-76.

Ozdemir, L., and Akdemir, N. (2009). Effects of multisensory stimulation on cognition, depression and anxiety levels of mildly-Alzheimer's patients. J. Neurol. Sci. 283, 211-213.

Parham,L.D., and Ecker,C. (2007). Sensory Processing Measure. Los Angeles, Western Psychological Services.

Pennington,B.F.(1991).Issuesin syndrome validation. In Diagnosing Learning Disorders: A Neuropsychological Framework, B. F. Pennington, ed. (New York, NY, The Guilford Press), pp. 23-31.
Piaget, J. (1952). The Origins of Intelligence in Children. New York, NY, International Universities Press.

Polatajko, H. J., Kaplan, B. J., and Wilson, B. N. (1992). Sensory integration treatment for children with learning disabilities: its status. 20 years later. Occup. Ther. J. Res. 12, 323-341.

Rogers, S. J., Hepburn, S., and Wehner, E. (2003). Parent reports of sensory symptoms in toddlers with autism and those with other developmental disorders. J. Autism Dev. Disord. 33, 631-642.

Rosenzweig, M. R. (1966). Environmental complexity, cerebral change, and behavior. Am. Psychol. 21, 321-332.

Ross, L. A., Saint-Amour, D., Leavitt, V.M., Javitt, D. G., and Foxe, J. J. (2007). Do you see what I am saying? Exploring visual enhancement of speech comprehension in noisy environments. Cereb. Cortex 17, 1147-1153.

Rowland, B., Stanford, T., and Stein, B. (2007). A Bayesian model unifies multisensory spatial localization with the physiological properties of the superior colliculus. Exp. Brain Res. 180, 153-161.

Schaaf, R. C., Miller, L. J., Sewell, D., and O'Keefe, S. (2003). Children with disturbances in sensory processing: a pilot study examining the role of the parasympathetic nervous system. Am. J. Occup. Ther. 57, 442-449.

Schaffer, R. (1984). Sensory integration therapy with learning disabled children: a critical review. Can. J. Occup. Ther. 51, 73-77.

Scheibel, M., and Scheibel, A. (1964). Some structural and functional substrates of development in young cats. Prog. Brain Res. 9, 6-25.

Schneider, M. L., Moore, C. F., Gajewski, L. L., Larson, J. A., Roberts, A. D., Converse, A. K., and DeJesus, O. T. (2008). Sensory processing disorder in a primate model: evidence from a longitudinal study of prenatal alcohol and prenatal stess effects. Child Dev. 79, 100-113.

Schneider, M. L., Moore, C. F., Gajewski, L. L., Laughlin, N. K., Larson, J.A., Gay, C. L., Roberts, A. D., Converse, A. K., and DeJesus, O. T. (2007). Sensory processing disorders in a nonhuman primate model: evidence of occupational therapy practice. Am. J. Occup. Ther. 61, 247-253.

Schoen, S. A., Miller, L. J., and Green, K. E. (2008). Pilot study of the sensory over-responsivity scales: assessment and inventory. Am. J. Occup. Ther. 62, 393-406.

Sherrington, C. (1955). Man in His Nature. Garden City, CO, Doubleday and Co.

Sherrington, C. S. (1906). The Integrative Action of the Nervous System. New Haven, CT, Yale University Press.

Solomon, P., Kubzansky, P. E. Leiderman, P. H., Mendelson, J. H., Trumball, R., and Wexler, D. (eds) (1961). Sensory Deprivation. Cambridge, MA, Harvard University.

Spittle, A. J., Orton, J., Doyle, L. W., and Boyd, R. (2007). Early developmental intervention programs post hospital discharge to prevent motor and cognitive impairments in preterm infants. Cochrane Database Syst. Rev. 18, CD005495.

Stein, B. E., Huneycutt, W. S., and Meredith, M. A. (1988). Neurons and behavior: the same rules of multisensory integration apply. Brain Res. 448 , 355-358.

Stein, B. E., and Meredith, M. A. (1993). The Merging of the Senses. Cambridge, MA, MIT Press.

Stein, B. E., Stanford, T. R., and Rowland, B. A. (2009). The neural basis of multisensory integration in the midbrain: its organization and maturation. Hear. Res. [Epub ahead of print].

Taft, L. (1972). Are we handicapping the handicapped? Dev. Med. Child Neurol. 14, 703-704.

Talsma, D., and Woldorff, M. G. (2005) Selective attention and multisensory integration: multiple phases of effects on the evoked brain activity. J. Cogn Neurosci. 17, 1098-1114.

Teder-Sälejärvi, W. A., Di Russo, F. McDonald, J. J., and Hillyard, S. A (2005). Effects of spatial congruity on audio-visual multimodal integration. J. Cogn. Neurosci. 17, 1396-1409.

Vargas, S., and Camilli, G. (1999). A meta-analysis of research on sensory integration treatment. Am. J. Occup. Ther. 53, 189-198.

Walker, K. F. (1993). A. Jean Ayres. In Perspectives on Theory for the Practice of Occupational Therapy, R. J. Miller and K. F. Walker, eds (Gaithersburg, MD, Aspen Publishers, Inc.), pp. 104-154.

Wallace, M. T. (2004). The development of multisensory integration. In The Handbook of Multisensory Processes, G. Calvert, C. Spence and B. E. Stein, eds (Cambridge, MA The MIT Press). pp. 625-642.
Wallace, M. T., Meredith, M. A., and Stein, B. E. (1992). Integration of multiple sensory modalities in cat cortex. Exp. Brain Res. 91, 484-488.

Wallace, M. T., Roberson, G. E., Hairston, W. D., Stein, B. E., Vaughan, J. W., and Schirillo, J. A. (2004). Unifying multisensory signals across time and space. Exp. Brain Res. 158, 252-258.

Wallace, M. T., and Stein, B. E. (2007). Early experience determines how the senses will interact. J. Neurophysiol. 97, 921-926.

Welch, R. B., and Warren, D.H. (1980). Immediate perceptual response to intersensory discrepancy. Psychol. Bull. 88, 638-667.

Welch, R. B., and Warren, D. H. (1986). Intersensory interactions. In Sensory Processes and Perception: Handbook of Perception and Performance, K.R. Boff, L. Kaufman and J.P. Thomas, eds (New York, NY, Wiley), pp. 1-36.

Westheimer, G., and McKee, S. P. (1977). Spatial configurations for visual hyperacuity. Vis. Res. 17, 941-947.

Wilson, B. N., Kaplan, B. J., Fellowes, S., Gruchy, C., and Faris, P. (1992). The efficacy of sensory integration treatment compared to tutoring. Phys. Occup. Ther. Pediatr. 12, 1-36.

World Health Organization (2004). International Statistical Classification of Diseases and Health Related Problems ICD-10, 10th edn. Geneva, World Health Organization.

Conflict of Interest Statement: The authors declare that the research was conducted in the absence of any commercial or financial relationships that could be construed as a potential conflict of interest.

Received: 02 April 2009; paper pending published: 27 April 2009; accepted: 03 September 2009; published online: 30 September 2009.

Citation: Miller LJ, Nielsen DM, Schoen SA and Brett-Green BA (2009) Perspectives on sensory processing disorder: a call for translational research. Front. Integr. Neurosci. 3:22. doi: 10.3389/neuro.07.022.2009

Copyright () 2009 Miller, Nielsen, Schoen and Brett-Green. This is an open-access article subject to an exclusive license agreement between the authors and the Frontiers Research Foundation, which permits unrestricted use, distribution, and reproduction in any medium, provided the original authors and source are credited. 